\title{
Serum copeptin level can be a helpful biomarker in evaluation of myocardial perfusion scintigraphy results
}

\author{
Hüseyin Ede ${ }^{1}$, Seyhan Karaçavuş ${ }^{2}$, Ayşe Yeşim Göçmen ${ }^{3}$, Barış Yaylak \\ Süleyman Akkaya ${ }^{4}$, Burak Açıkgöz ${ }^{4}$, Neslihan Çilek ${ }^{2}$, Mahmut Kılıç ${ }^{5}$, Ali Rıza Erbay ${ }^{1}$ \\ ${ }^{1}$ Department of Cardiology, Faculty of Medicine, Bozok University, Yozgat, Turkey \\ ${ }^{2}$ Department of Nuclear Medicine, Faculty of Medicine, Bozok University, Yozgat, Turkey \\ ${ }^{3}$ Department of Biochemistry, Faculty of Medicine, Bozok University, Yozgat, Turkey \\ ${ }^{4}$ Cardiology Clinic, Diyarbakır Gazi Yaşargil Education and Research Hospital, Diyarbakır, Turkey \\ ${ }^{5}$ Department of Public Health, the School of Health, Bozok University, Yozgat, Turkey
}

\begin{abstract}
Background: Myocardial perfusion scintigraphy (MPS) is a well-established diagnostic tool. The sensitivity and specificity of single photon emission computed tomography (SPECT) MPS to detect significant coronary lesion were $86 \%$ and $74 \%$, respectively. The aim of this study was to examine the role of serum copeptin in evaluation of MPS.

Methods: Sixty-two consecutive patients underwent both SPECT MPS using ${ }^{99 m}$ Tc-sestamibi and transthoracic echocardiography were enrolled prospectively. Age, gender, height, weight, presence of cardiovascular risk factors were recorded. Exercise treadmill test (ETT) with modified Bruce protocol was used to induce coronary ischemia during MPS. While performing $M P S$, blood samples for serum copeptin level were drawn three times at pre-exercise, at the peak of ETT, and $6 \mathrm{~h}$ after ETT, respectively. The patients were enrolled into three groups according to MPS results (normal, equivocal and ischemia).
\end{abstract}

Results: The study included 62 patients (23 with normal, 20 with equivocal, 19 with ischemia on MPS). Pre-, peak-, and post-exercise B-type natriuretic peptide and troponin I values were similar across the groups ( $p>0.05$ for all comparisons). Serum copeptin values for pre-and peak-exercise were similar among all groups $(p=0.883$ and $p=0.089)$. Post-exercise copeptin values of the normal and equivocal groups were similar ( $p=0.661, z=-0.438)$ while that of the ischemia group was significantly higher than both the normal $(p<0.001)$ and equivocal group $(p<0.001)$.

Conclusions: Serum copeptin was found to be increasing significantly in case of ischemia on MPS. It may be used in differentiation of equivocal results from false positive results. (Cardiol J 2016; 23, 1: 71-77)

Key words: copeptin, myocardial perfusion scintigraphy, coronary artery disease, ischemia

Address for correspondence: Assistant Prof. Dr Hüseyin Ede, Bozok University, Faculty of Medicine, Cardiology Department, Adnan Menderes, Bulvarı No: 4466200 Yozgat, Turkey, tel: 0090 5056711104, 00903542127060 , fax: 00903542123739 , e-mail: huseyinede@gmail.com

Received: 28.04.2015

Accepted: 25.05.2015 


\section{Introduction}

Myocardial perfusion scintigraphy (MPS) is a well-established diagnostic tool in the differentiation of new-onset chest pain of patients with or without previous coronary artery disease (CAD). MPS is especially helpful for the patients with basal electrocardiographic (ECG) changes as left bundle branch block, left ventricle (LV) hypertrophy, pre-excitation and with non-diagnostic exercise ECG test results, or for subjects unable to exercise due to orthopedic or neurological problems [1].

The sensitivity and specificity of single photon emission computed tomography (SPECT) MPS to detect significant coronary lesion (defined as coronary stenosis of more than $50 \%$ ) were $86 \%$ and $74 \%$, accordingly [2]. A false negative result can be obtained in the SPECT MPS due to balanced multiple vessel disease. Thus, nearly $13-15 \%$ of patients with left main disease can have normal perfusion scintigraphy due to balanced ischemia in multivessel CAD [3, 4]. Other disadvantages in MPS are equivocal results due to attenuation artifacts, patient positional movement during the test, or incorrectly applied technical analyses. Also incorrectly performed technical analyses such as multidetector misallignment, incorrectly designed bull's eye reconstruction, presence of non-uniform flood fields can lead to false positive or equivocal results [5]. Guided by these results, physicians can be forced to apply invasive diagnostic tests. In such false positive cases, application of gated studies, attenuation correction algorithms, and prone imaging technique can promote diagnostic accuracy but cannot be enough to solve the problem all the time [6-8].

Copeptin, the C-terminal part of the vasopressin prohormone, is released stoichiometrically with arginine-vasopressin from the neurohypophysis and seems to reflect the individual endogenous stress level and also the mortality risk in coronary events [9]. As the endogenous stress is already present at the onset of coronary ischemia, copeptin appears to be able to detect coronary ischemia very early following symptom onset, even when cardiac troponin is still negative [10]. The role of copeptin in evaluation of MPS results has not been studied extensively.

The aim of our study was to search role of serum copeptin in further evaluation of different myocardial perfusion scintigraphy results (normal scan, equivocal, and ischemia) among patients in suspect of CAD.

\section{Methods}

\section{Subjects}

We prospectively studied 62 consecutive patients referred to the Cardiology Department with chest pain in suspicion of CAD. The patients underwent both SPECT MPS using technetium-99msestamibi $\left({ }^{99 \mathrm{~m}} \mathrm{Tc}\right.$-sestamibi) and transthoracic echocardiography between January 2014 and September 2014. Subjects aged 18-65 years old were included in the study.

Patients with previous CAD, acute coronary syndrome, angina pectoris of Canadian Cardiology Society class III or IV angina, peripheric vascular disease, chronic kidney disease (creatinine $>1.4 \mathrm{mg} /$ $/ \mathrm{dL}$ ), advanced liver disease, stroke, cancer, infection, hyper- or hypothyroidism, symptomatic congestive heart failure, left ventricular ejection fraction (LVEF) less than 50\%, any congenital heart disease were excluded. In addition, patients with history of myocardial infarction based on echocardiography and ECG findings or coronary artery bypass grafting were also ruled out.

The following data were also obtained: age, gender, height, weight, presence of cardiovascular risk factors. Cardiovascular risk factors were determined according to the following criteria: positive family history for CAD (presence of CAD in first-degree family members, male $<55$ years and/ /or female $<65$ years), cigarette smoking (current smoking or smoking in the last 2 years), hypertension (the last 3 blood pressure measurements $>140 / 90 \mathrm{~mm} \mathrm{Hg}$ or treatment with antihypertensive medication within the last 6 months), hyperlipidemia (current usage of cholesterol-lowering medication).

Body mass index (BMI) was defined according to the World Health Organization criteria. Patients were classified as normal weight (BMI 18.5$-24.9 \mathrm{~kg} / \mathrm{m}^{2}$ ), overweight (BMI $25.0-29.9 \mathrm{~kg} / \mathrm{m}^{2}$ ), obese class I (BMI $\left.30.0-34.9 \mathrm{~kg} / \mathrm{m}^{2}\right)$, obese class II (BMI $35.0-39.9 \mathrm{~kg} / \mathrm{m}^{2}$ ) or obese class III (BMI $40 \mathrm{~kg} / \mathrm{m}^{2}$ or more).

The ethical approval and informed consent from each patient was provided for the study and the investigation was performed in accordance with the principles outlined in the Declaration of Helsinki.

\section{Rest/stress myocardial perfusion scintigraphy protocol \\ In this study, we used a same-day stress/rest protocol, with the stress test performed in the}


morning between 08:00-10:00 a.m. and the rest test performed on same day $6 \mathrm{~h}$ after the stress test. All subjects underwent exercise treadmill test (ETT) with modified Bruce protocol to induce coronary ischemia. End points of exercise testing were physical exhaustion, severe angina, sustained ventricular arrhythmia or exertional hypotension. At near-maximal exercise, ${ }^{99 \mathrm{~m}} \mathrm{Tc}$-sestamibi was injected and exercise was continued for two additional 2 min after injection. The patients who could not achieve at least 6 -min exercise and $85 \%$ of targeted heart rate (220 min age in years) were excluded from the study.

\section{SPECT acquisition protocol}

The SPECT data were acquired with Gated technique using the double-head SPECT gammacamera system (Philips Medical Systems Brightview Gamma Diagnostic, Holland) equipped with a high-resolution low-energy collimator. A total of 32 projections (35 s/projection) were obtained over a $180^{\circ}$ circular orbit, at the $20 \%$ energy window which centered $140 \mathrm{keV}$ for gamma emission of technetium-99m. Myocardial images were projected into tomographic slices in the short axis, verticallong axis and horizontal-long axis views. 4D-M SPECT software was used for semiquantitative analysis of data. The SPECT images were reconstructed by filtered back projection method using a Butterworth filter (order 5; cut-off frequency 0.50).

\section{Image interpretation}

The perfusion images were evaluated independently by two experienced nuclear medicine physicians without clinical data. Disagreement was solved by consensus. The myocardium was divided into 17 segments for semiquantitative analysis, following the American Society of Nuclear Cardiology, the American College of Cardiology and the American Heart Association guidelines [11]. A scale of 0-4 was used for grading wall motion: $(0$ - normal, 1 - mildly hypokinetic, 2 - hypokinetic, 3 - akinetic, and 4-dyskinetic) by automatic scores for each of the segments [12]. Abnormal motion was defined as a score of $>2$. According to the test results, patients were classified into three groups. Normal group was the patients with normal Gated SPECT MPS scans. Equivocal group included indeterminate scan results that patients with slight perfusion and/or mildly hypokinetic wall motion. Third group was the ischemia group including patients with apparent abnormal perfusion and/or wall motion findings in any segment of LV myocardium at stress.

\section{Echocardiography protocol}

Two-dimensional (2D), M-mode, pulsed Doppler and tissue Doppler echocardiography were performed on an ultrasound machine (Presound alpha 7, IPF 1701 Model, 2009; Hitachi Aloka Medical, Ltd. Tokyo, Japan) with a $2.5-\mathrm{MHz}$ transducer by cardiologist blinded to the study before performing MPS. Standard 2D measurements (LV diastolic and systolic dimension, ventricular septum and posterior wall thickness, left atrial diameter, LVEF) were obtained as recommended by the American Society of Echocardiography [13]. Mitral annular velocities were obtained by Doppler tissue imaging using the pulsed-wave mode. Early diastolic mitral annular (Em), late diastolic (Am) and systolic velocities $(\mathrm{Sm})$ of the mitral annulus were measured from the apical 4-chamber view with a 2 - to $5-\mathrm{mm}$ sample volume placed at the lateral edge of the mitral annulus. All measurements were carried out at expirium.

\section{Biochemical measurements}

A specimen of venous blood was drawn before (pre-exercise), 1 min after peak exercise (peak-exercise) and $6 \mathrm{~h}$ after exercise (post-exercise) in the seated position from a catheter inserted into the antecubital vein. These samples were collected in plastic tubes containing ethylenediaminetetraacetic acid (EDTA). They were placed on ice and then centrifuged at 3,000 g; and plasma was frozen at $-80^{\circ} \mathrm{C}$ until assay. Commercial enzymelinked immunosorbent assay (ELISA) kits were used to measure Human B-type natriuretic peptide (Ray Biotech, Inc. USA), cardiac troponin I (cTnI) (Ray Biotech, Inc. USA), human copeptin (ELABSCIENCE, Wuhan, Hubei Province, China). The laboratory technician who measured copeptin was at a different place and blinded to the data of the patients. All other parameters were measured using standard laboratory methods in the core laboratory. Serum levels of high density lipoprotein (HDL), low density lipoprotein (LDL), total cholesterol, triglyceride (TG), creatinine, thyroid stimulating hormone (TSH) and fasting blood glucose (FBG) were measured for all patients accordingly.

\section{Statistical analysis}

Statistical analyses were performed using the SPSS software version 18. Continuous variables are presented as mean \pm standard deviation and categorical variables are presented as frequencies (\%). Except hemoglobin, creatinine, total cholesterol, and LDL, all other continuous variables did not have normal distribution according to 
Table 1. Demographic and clinical data of the subjects.

\begin{tabular}{lcccc}
\hline & $\begin{array}{c}\text { Normal group } \\
(\mathbf{n}=\mathbf{2 3})\end{array}$ & $\begin{array}{c}\text { Equivocal group } \\
(\mathbf{n}=\mathbf{2 0})\end{array}$ & $\begin{array}{c}\text { Ischemia group } \\
\text { (n = 19) }\end{array}$ & $\mathbf{P}$ \\
\hline Age [year] & $52 \pm 12$ & $48 \pm 10$ & $54 \pm 10$ & 0.161 \\
Gender (F/M) & $17 / 6(74 / 26)$ & $12 / 8(60 / 40)$ & $11 / 8(58 / 42)$ & 0.489 \\
Height [cm] & $162 \pm 4$ & $161 \pm 4$ & $164 \pm 3$ & 0.100 \\
Weight [kg] & $71 \pm 3$ & $71 \pm 4$ & $72 \pm 6$ & 0.511 \\
Body mass index & $27.1 \pm 1.7$ & $27.5 \pm 1.6$ & $27.0 \pm 2.2$ & 0.445 \\
Diabetes mellitus & $7(30 \%)$ & $4(205)$ & $3(16 \%)$ & 0.499 \\
Hypertension & $8(35 \%)$ & $2(10 \%)$ & $6(32 \%)$ & 0.142 \\
Cigarette smoking & $4(17 \%)$ & $6(30 \%)$ & $5(26 \%)$ & 0.608 \\
Family history of premature CAD & $5(22 \%)$ & $3(15 \%)$ & $4(215 \%)$ & 0.834 \\
Hyperlipidemia & $1(4 \%)$ & $0(0 \%)$ & $3(15 \%)$ & 0.117 \\
Systolic BP [mm Hg] & $120 \pm 11$ & $121 \pm 10$ & $127 \pm 11$ & 0.169 \\
Diastolic BP [mm Hg] & $76 \pm 4$ & $77 \pm 3$ & $75 \pm 4$ & 0.279 \\
Exercise duration [s] & $600 \pm 140$ & $651 \pm 124$ & $616 \pm 155$ & 0.553 \\
Rate pressure product at peak & $25,805 \pm 4,413$ & $25,502 \pm 5,101$ & $27,147 \pm 4,775$ & 0.467 \\
exercise [mm Hg/min] & $10.8 \pm 2.11$ & $11.2 \pm 2.0$ & $11.0 \pm 2.2$ & 0.847 \\
Maximal workload [MET] & & &
\end{tabular}

$\mathrm{BP}$ - blood pressure; CAD — coronary artery disease; $\mathrm{F}$ - female; $\mathrm{M}$ - male; MET — metabolic equivalent

Kolmogorov-Smirnov test. Categorical variables were compared using the $\chi^{2}$ test. Spearman simple correlation analyses were performed to determine the association between continuous parameters accordingly while Mann-Whitney U test and Kruskal-Wallis test were used to compare groups accordingly. Wilcoxon rank-sum test was used to compare pre-, peak- and post-exercise biochemical values within the groups. To find diagnostic cut-off value of copeptin for differentiation of patients with normal scan from patients with ischemia, a receiver operating characteristic (ROC) curve analysis was constructed, and the area under the curve (AUC) was reported, which is considered representative of the discriminative ability of the variable cut-off. Sensitivity and specificity values of the best cutoff variables were determined using ROC curve analysis. A p value of less than 0.05 was considered to show statistically significant result.

\section{Results}

Sixty-two subjects were included in the study and the data of the patients undergone to rest/ /treadmill stress myocardial perfusion SPECT were expressed in Table 1 . At rest/treadmill myocardial perfusion SPECT, an ischemia was induced in $19(31 \%)$ patients, while $23(37 \%)$ patients had normal MPS results and $20(32 \%)$ patients with equivocal test results. All subjects succeeded at least 6-min exercise and $85 \%$ of targeted heart rate. Endpoint of the exercise test was physical exhaustion for all patients except 1 patient who described angina without relevant ECG changes. The data related to treadmill test findings were summarized in Table 1. All groups had statistically similar LVEF, left atrial diameter and $\mathrm{Em} / \mathrm{Am}$ ratios. Laboratory findings of serum FBG, TSH, creatinine and fasting lipid profile were statistically similar among the groups (Table 2 ).

Subjects in the equivocal group were further evaluated in suspect of CAD accordingly. We found that 11 patients had normal coronary arteries according to result of conventional coronary angiography (CAG) and 4 patients had zero-score coronary computed tomographic angiography result, 3 patients had non-obstructive and 2 patients with obstructive CAD upon CAG or computed tomography examinations.

In the ischemia group, $6(32 \%)$ patients had apparent abnormal perfusion and/or wall motion findings at inferior wall, 7 (37\%) patients at inferolateral, $5(26 \%)$ patients at anterior wall, $1(5 \%)$ patients at apical part of the LV. $89 \%$ of subjects with ischemia on MPS $(\mathrm{n}=18)$ revealed obstructive $\mathrm{CAD}$ according to the CAG results. Two subjects had non-obstructive CAD.

Median serum copeptin levels increased significantly with exercise in the all subjects from 7.8 (IQR 7.2-8.1) to 9.7 (IQR 8.7-10.6) pmol/L 
Table 2. Laboratory findings of the subjects.

\begin{tabular}{lcccc}
\hline & $\begin{array}{c}\text { Normal group } \\
(\mathbf{n}=\mathbf{2 3})\end{array}$ & $\begin{array}{c}\text { Equivocal group } \\
(\mathbf{n}=\mathbf{2 0})\end{array}$ & $\begin{array}{c}\text { Ischemia group } \\
(\mathbf{n}=\mathbf{1 9 )}\end{array}$ & $\mathbf{P}$ \\
\hline Fasting blood glucose $[\mathrm{mg} / \mathrm{dL}]$ & $110 \pm 29$ & $108 \pm 34$ & $112 \pm 35$ & 0.425 \\
Creatinine $[\mathrm{mg} / \mathrm{dL}]$ & $0.82 \pm 0.19$ & $0.86 \pm 0.12$ & $0.86 \pm 0.13$ & 0.334 \\
Total cholesterol $[\mathrm{mg} / \mathrm{dL}]$ & $193 \pm 24$ & $189 \pm 24$ & $194 \pm 26$ & 0.515 \\
Triglyceride $[\mathrm{mg} / \mathrm{dL}]$ & $155 \pm 60$ & $161 \pm 57$ & $164 \pm 56$ & 0.724 \\
High density lipoprotein $[\mathrm{mg} / \mathrm{dL}]$ & $47 \pm 8$ & $43 \pm 10$ & $42 \pm 6$ & 0.161 \\
Low density lipoprotein $[\mathrm{mg} / \mathrm{dL}]$ & $116 \pm 22$ & $115 \pm 17$ & $121 \pm 20$ & 0.416 \\
Thyroid stimulating hormone $[\mathrm{IU} / \mathrm{L}]$ & $1.75 \pm 0.74$ & $1.85 \pm 1.28$ & $1.54 \pm 0.75$ & 0.621 \\
\hline
\end{tabular}

Table 3. Echocardiographic and biochemical measurements of the subjects.

\begin{tabular}{lcccc}
\hline & $\begin{array}{c}\text { Normal group } \\
(\mathbf{n}=\mathbf{2 3})\end{array}$ & $\begin{array}{c}\text { Equivocal group } \\
(\mathbf{n}=\mathbf{2 0})\end{array}$ & $\begin{array}{c}\text { Ischemia group } \\
(\mathbf{n}=\mathbf{1 9})\end{array}$ & $\mathbf{P}$ \\
\hline Left ventricular ejection fraction [\%] & $62 \pm 3$ & $64 \pm 3$ & $62 \pm 2$ & 0.061 \\
Left atrium diameter [mm] & $37 \pm 3$ & $37 \pm 2$ & $39 \pm 2$ & 0.070 \\
Mitral annulus TDI Em/Am & $1.48 \pm 0.78$ & $1.66 \pm 0.60$ & $1.34 \pm 0.66$ & 0.364 \\
Pre-exercise BNP [pg/mL] & $10.0 \pm 1.8$ & $9.4 \pm 1.6$ & $10.3 \pm 3.1$ & 0.467 \\
Peak-exercise BNP [pg/mL] & $14.5 \pm 5.5$ & $11.3 \pm 1.9$ & $12.5 \pm 3.8$ & 0.078 \\
Post-exercise BNP [pg/mL] & $9.0 \pm 1.3$ & $8.3 \pm 1.2$ & $9.2 \pm 2.4$ & 0.330 \\
Pre-exercise troponin I [ng/mL] & $0.064 \pm 0.008$ & $0.064 \pm 0.007$ & $0.062 \pm 0.005$ & 0.712 \\
Peak-exercise troponin I [ng/mL] & $0.085 \pm 0.012$ & $0.082 \pm 0.014$ & $0.084 \pm 0.011$ & 0.884 \\
Post-exercise troponin I [ng/mL] & $0.070 \pm 0.016$ & $0.066 \pm 0.009$ & $0.074 \pm 0.016$ & 0.296 \\
Pre-exercise copeptin [pmol/L] & $7.7 \pm 0.7$ & $7.7 \pm 0.6$ & $7.8 \pm 0.7$ & 0.883 \\
Peak-exercise copeptin [pmol/L] & $9.2 \pm 1.5$ & $9.5 \pm 1.8$ & $10.5 \pm 1.8$ & 0.089 \\
Post-exercise copeptin [pmol/L] & $7.7 \pm 2.7$ & $7.8 \pm 2.5$ & $12.2 \pm 2.0$ & 0.000 \\
$\Delta$ Copeptin [pmol/L] & $0.02 \pm 2.55$ & $0.16 \pm 2.30$ & $4.40 \pm 2.02$ & 0.000 \\
\hline
\end{tabular}

BNP — B-type natriuretic peptide; TDI — tissue Doppler imaging

$(\mathrm{p}<0.001)$. However, median serum copeptin level of patients with ischemia on MPS increased significantly with exercise from 7.8 (IQR 7.2-8.4) to 10.9 (IQR 8.8-12.1) pmol/L ( $<0.001)$ and that of patients with normal result on MPS from 7.7 (IQR 7.0-8.1) to 9.4 (IQR 8.2-10.3) pmol/L ( $<<0.005$ ). Similarly, serum copeptin level of patients with equivocal test results on MPS increased significantly with exercise from 7.8 (IQR 7.4-8.0) to 9.6 (IQR 8.7-10.1) pmol/L ( $<0.005)$.

Median serum post-exercise copeptin levels of patients with normal and equivocal test results on MPS were returned to pre-exercise levels while that of patients with ischemia on MPS increased more compared to their pre-exercise levels (Table 3). Cardiac troponin and serum B-type natriuretic peptide levels did not show any significant change with exercise.
Average pre-exercise copeptin level did not differ significantly between man and women $(7.7 \pm$ \pm 0.6 and $7.7 \pm 0.8 \mathrm{pmol} / \mathrm{L}$, respectively, $\mathrm{p}=0.622$ ). Additionally, we did not find any statistical correlation of pre-exercise copeptin level and age $(\mathrm{r}=-0.208, \mathrm{p}=0.105)$.

ROC curve analysis was conducted to find cutoff value for serum copeptin to detect myocardial ischemia. Cut-off value for post-exercise serum copeptin was $9.83 \mathrm{pmol} / \mathrm{L}$ with sensitivity of $94.7 \%$ and specificity of $73.9 \%$ (AUC 0.918 with $95 \% \mathrm{CI}$ $0.837-0.999$ and $\mathrm{p}<0.001)$. ROC curve analysis was also performed for $\Delta$ copeptin (the difference of post-exercise copeptin from pre-exercise copeptin) to detect myocardial ischemia. Cut-off value for $\Delta$ copeptin was found to be $1.85 \mathrm{pmol} / \mathrm{L}$ with sensitivity of $89.5 \%$ and specificity of $73.9 \%$ (AUC 0.924 with 95\% CI 0.850-0.999, $\mathrm{p}<0.001$ ). 


\section{Discussion}

This study prospectively explored the role of copeptin in evaluation of myocardial perfusion test results among patients referred for treadmill exercise myocardial perfusion SPECT. We can summarize our findings in 4 major headings. Firstly, serum copeptin levels increase significantly with exercise, independent of myocardial perfusion results. Secondly, response of patients with normal or equivocal perfusion images to exercise was similar in respect to serum pre-, peak-, and post-exercise copeptin levels. Thirdly, patients with ischemia on MPS had significantly higher post-exercise copeptin level compared to the others. Fourth, post-exercise serum copeptin level and $\Delta$ copeptin (the difference between pre- and post-exercise) does seem beneficial biomarker in detection of myocardial ischemia on MPS rather than the difference between pre- and peak-exercise copeptin levels.

Vasopressin, also known as antidiuretic hormone, is a nanopeptide produced in the hypothalamus and secreted into the circulation from the neurohypophysis to maintain osmolarity and water homeostasis [14]. Copeptin is the C-terminal part of the vasopressin prohormone. Opposite to vasopressin which has a half-life of $10-20 \mathrm{~min}$, copeptin can be detected in the circulation for days [15]. Serum vasopressin level has been shown to be increased in heart failure, septic shock, myocardial infarction and other endogenous stress conditions [16-18].

Results from our study were consistent with previous studies searching response of vasopressin and copeptin to exercise. It was reported that serum copeptin was increased in response to exercise, proportional to increment in workload [15, 19]. In our study, exercise statistically elevated serum copeptin levels at peak exercise in all subjects irrespective of myocardial perfusion results. This finding was in concordant with the literature reporting increment of vasopressin in proportion to a 6 -min walk test result among subjects with known CAD [20]. Mockel and Searle [21] also confirmed this finding. They found that serum copeptin level increased significantly in subjects with both normal and ischemia on MPS. However, both groups had similar peak-baseline copeptin difference during exercise [21].

In our study, we also found that the subjects with normal and ischemic MPS result had similar peak-baseline copeptin changes $(1.5 \pm 1.7$ vs. $2.8 \pm 1.9 \mathrm{pmol} / \mathrm{L}, \mathrm{p}=0.063)$. In addition to the study by Mockel and Searle [21], we followed the subjects and measured serum copeptin level $6 \mathrm{~h}$ after the treadmill test. We found that post-exercise copeptin level of subjects with ischemia on MPS had significantly higher than the others, while values of subjects with normal and equivocal test results returned to pre-exercise levels. In parallel to these findings, it was revealed that $\Delta$ copeptin value of ischemia group was significantly higher than that of normal and equivocal groups. We concluded that additional endogenous stress due to coronary ischemia led further increment in post-exercise copeptin levels. This finding can be very beneficial especially in false negative cases such as normal perfusion scintigraphy due to balanced ischemia in multivessel CAD [3, 4]. Confirmation of increased copeptin level at post-exercise can guide physician accordingly to apply further diagnostic interventions.

Copeptin has been shown to be a helpful marker in detection of acute myocardial infarction and prognostic marker in heart failure with low ejection fraction [21,22]. There have been studies searching the use of copeptin in differentiation of exercise-induced ischemia on MPS but our study investigated for the first time the behavior of copeptin both during and after the exercise [23]. According to our results, neither pre-exercise nor peak-exercise copeptin levels was statistically related to ischemia on MPS but post-exercise copeptin level and $\Delta$ copeptin revealed to be an indicator for detection of ischemia on perfusion images.

Another aim of our study was to differentiate patients with normal and equivocal MPS results. Equivocal test results are frequently encountered in clinical practice and can lead physician to apply further non-invasive and invasive diagnostic tests. Although several technical and patient related interventions have been introduced to handle this problem, but none of them has ability to solve the problem totally [5-8]. Here, we introduced another aspect of copeptin use. We found that patients with equivocal perfusion images had similar pattern of serum copeptin changes in response to exercise. In other words, detection of post-exercise copeptin level reduction into pre-exercise level may be helpful sign to interpret equivocal perfusion images as normal MPS result.

Neither adenosine nor dipyridamole was used to induce coronary ischemia, Instead, we used ETT. Adenosine based MPS was proven to have higher diagnostic ability than dipyridamole based protocols, however it had equal diagnostic ability compared to exercise based protocols $[24,25]$. Thus, to obtain measurable and similar level of physical stress, we applied ETT to induce coronary ischemia. 


\section{Limitations of the study}

Number of subjects with ischemia on MPS was low, thus we could not perform any further analysis for relation between extent of myocardial ischemia and post-exercise copeptin increment. Failure to exclude possible presence of CAD in all patients with equivocal MPS was another limitation to the study.

\section{Conclusions}

Copeptin is a rapidly detected biomarker, it can be detected in the circulation even before troponin becomes positive. It increases in presence of acute myocardial infarction, heart failure, shock, and endogenous stress. Here, we have proven that patients with normal and equivocal MPS results had similar pattern of copeptin changes in response to exercise, while patients with ischemia on MPS had higher level of post-exercise copeptin and $\Delta$ copeptin levels. Copeptin may be helpful both to detect ischemia and to differentiate equivocal test results on MPS.

\section{Acknowledgements}

The study was supported by Bozok University Scientific Research Department (Project No: 2014TF/A94).

\section{Conflict of interest: None declared}

\section{References}

1. Fihn SD, Gardin JM, Abrams J et al. 2012 ACCF/AHA/ACP/ /AATS/PCNA/SCAI/STS Guideline for the diagnosis and management of patients with stable ischemic heart disease: A report of the American College of Cardiology Foundation/American Heart Association Task Force on Practice Guidelines, and the American College of Physicians, American Association for Thoracic Surgery, Preventive Cardiovascular Nurses Association, Society for Cardiovascular Angiography and Interventions, and Society of Thoracic Surgeons. J Am Coll Cardiol, 2012; 60: e44-e164.

2. Pakkal M, Raj V, McCann GP. Non-invasive imaging in coronary artery disease including anatomical and functional evaluation of ischaemia and viability assessment. Br J Radiol, 2011; 84: S280-S295.

3. Melikian N, De Bondt P, Tonino P et al. Fractional flow reserve and myocardial perfusion imaging in patients with angiographic multivessel coronary artery disease. J Am Coll Cardiol Intv, 2010; 3: 307-314.

4. Berman DS, Kang X, Slomka PJ et al. Underestimation of extent of ischemia by gated SPECT myocardial perfusion imaging in patients with left main coronary artery disease. J Nucl Cardiol, 2007; 14: 521-528.

5. DePuey EG 3rd. How to detect and avoid myocardial perfusion SPECT artifacts. J Nucl Med, 1994; 35: 699-702.

6. Ben-Haim S, Almukhailed O, Neill J et al. Clinical value of supine and upright myocardial perfusion imaging in obese patients using the D-SPECT camera. J Nucl Cardiol, 2014; 21: 478-485.
7. Karacavus S, Celik A, Tutus A, Kula M, Oguzhan A, Kalay N. Can left ventricular parameters examined by gated myocardial perfusion scintigraphy and strain echocardiography be prognostic factors for major adverse cardiac events? Hell J Nucl Med, 2014; 17: 10-11.

8. Worden NE, Lindower PD, Burns TL, Chatterjee K, Weiss RM. A second look with prone SPECT myocardial perfusion imaging reduces the need for angiography in patients at low risk for cardiac death or MI. J Nucl Cardiol, 2015; 22: 115-122.

9. Mueller C. Biomarkers and acute coronary syndromes: An update. Eur Heart J, 2014; 35: 552-556.

10. Lipinski MJ, Escárcega RO, D’Ascenzo $\mathrm{F}$ et al. A systematic review and collaborative meta-analysis to determine the incremental value of copeptin for rapid rule-out of acute myocardial infarction. Am J Cardiol, 2014; 113: 1581-1591.

11. Cerqueira MD, Weissman NJ, Dilsizian V et al. Standardized myocardial segmentation and nomenclature for tomographic imaging of the heart. A statement for healthcare professionals from the Cardiac Imaging Committee of the Council on Clinical Cardiology of the American Heart Association. Circulation, 2002; 105: 539-542.

12. Ficaro EP, Lee BC, Kritzman JN, Corbett JR. Corridor DM. The Michigan method for quantitative nuclear cardiology. J Nucl Cardiol, 2007; 14: 455-465.

13. Lang RM, Bierig M, Devereux RB et al. Chamber Quantification Writing Group, American Society of Echocardiography's Guidelines and Standards Committee, European Association of Echocardiography.Recommendations for chamber quantification: A report from the American Society of Echocardiography's Guidelines and StandardsCommittee and the Chamber Quantification Writing Group, developed in conjunction with the European Association of Echocardiography, a branch of the European Society of Cardiology. J Am Soc Echocardiogr, 2005; 18: 1440-1463.

14. Singh Ranger G. The physiology and emerging roles of antidiuretic hormone. Int J Clin Pract, 2002; 56: 777-782.

15. Morgenthaler NG, Struck J, Alonso C, Bergmann A. Assay for the measurement of copeptin, a stable peptide derived from the precursor of vasopressin. Clin Chem, 2006; 52: 112-119.

16. Finley IV JJ, Konstam MA, Udelson JE. Arginine vasopressin antagonist for the treatment of heart failure and hyponatremia. Circulation, 2008; 118: 410-421.

17. Jochberger S, Morgenthaler NG, Mayr VD et al. Copeptin and arginine vasopressin concentrations in critically ill patients. J Clin Endocrinol Metab, 2006; 91: 4381-4386.

18. Dunser MW, Mayr AJ, Ulmer $\mathrm{H}$ et al. Arginine vasopressin in advanced vasodilatory shock: A prospective, randomized, controlled study. Circulation, 2003; 107: 2313-2319.

19. Wade CE, Claybaugh JR. Plasma renin activity, vasopressin concentration, and urinary excretory responses to exercise in men. J Appl Physiol, 1980; 49: 930-936.

20. Radke KJ, King KB, Blair ML, Fitzpatrick PG, Eldredge DH. Hormonal responses to the 6-minute walk test in women and men with coronary heart disease: A pilot study. Heart Lung, 2005; 34: 126-135.

21. Möckel M, Searle J. Copeptin-marker of acute myocardial infarction. Curr Atheroscler Rep, 2014; 16: 421.

22. Pozsonyi Z, Förhécz Z, Gombos T, Karádi I, Jánoskuti L, Prohászka $Z$. Copeptin (C-terminal pro arginine-vasopressin) is an independent long-term prognostic marker in heart failure with reduced ejection fraction. Heart Lung Circ, 2015; 24: 359-367.

23. Staub D, Morgenthaler NG, Buser C et al. Use of copeptin in the detection of myocardial ischemia. Clin Chim Acta, 2009; 399: 69-73.

24. Conti A, Mariannini Y, Canuti E et al. Nuclear scan strategy and outcomes in chest pain patients value of stress testing with dipyridamole or adenosine. World J Nucl Med, 2014; 13: 94-101.

25. Chen GB, Wu H, He XJ et al. Adenosine stress thallium-201 myocardial perfusion imaging for detecting coronary artery disease at an early stage. J Xray Sci Technol, 2013; 21: 317-322. 